\section{Factores asociados al sedentarismo e inactividad física en Chile: una revisión sistemática cualitativa}

\author{
JORGE AHUMADA TELLO ${ }^{1, \mathrm{a}}$, MARÍA CECILIA TOFFOLETTO²,b
}

\section{Factors associated with sedentary lifestyle and physical inactivity in Chile: a qualitative systematic review}

\begin{abstract}
Background: Sedentary lifestyle and physical inactivity in Chile, has become a major problem and a public health priority in the last decade, as it increases the risk of non-communicable diseases. Aim: To identify which factors are associated with sedentary lifestyle and physical inactivity in Chile. Material and Methods: Qualitative systematic review of papers published between 2009-2019 in BIREME, SCIELO, WoS, Scopus and Medline databases, retrieving 29,752 and leaving finally five articles for review. Results: The main risk factors associated with sedentary lifestyle (according to the Chilean National Health Survey) were: living in an urban area, high income levels, being smoker or ex-smoker, being physically inactive, having a DVD, computer or notebook at home and a motorized vehicle. Physical inactivity was associated with an age $\geq 65$ years, hypertension and sedentary lifestyle. There are some common factors such as female sex, obesity, type 2 diabetes mellitus and metabolic syndrome. Conclusions: here are independent factors associated with both sedentary lifestyle and physical inactivity in Chile. Some factors are common for the two unhealthy behaviors such as female gender, obesity, type 2 diabetes mellitus and metabolic syndrome.
\end{abstract}

(Rev Med Chile 2020; 148: 233-241)

Key words: Risk Factors; Sedentary Behavior; Social Determinants of Health.

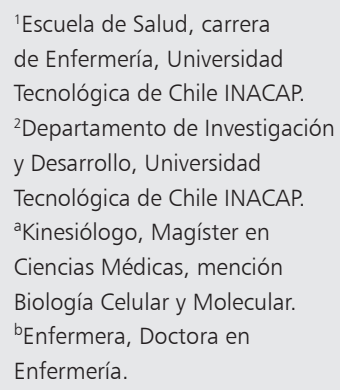

Trabajo no recibió financiamiento. Los autores declaran no tener conflictos de interés.

Recibido el 19 de julio de 2019 aceptado el 21 de noviembre de 2019.

Correspondencia a: María Cecilia Toffoletto Avenida Cachapoal, 370, casa 01, Rancagua. mtoffoletto@inacap.cl

\section{L}

as conductas sedentarias incluyen sentarse durante los desplazamientos, en el lugar de trabajo y el entorno doméstico durante el tiempo libre. Los comportamientos sedentarios como ver televisión, usar la computadora o sentarse en un automóvil generalmente están en el rango de gasto de energía de 1,0 a 1,5 múltiplos de la tasa metabólica basal (MET). Por su parte, la inactividad física se define como no cumplir con las pautas de actividad física recomendadas por entidades internacionales, esto es: $150 \mathrm{~min}$ de actividad física moderada a vigorosa por semana ${ }^{1,2}$.

En el informe de 2010 sobre el Estado de las Enfermedades no Transmisibles (ENT), la Organización Mundial de la Salud (OMS) estimó que,
3,2 millones de personas mueren cada año en consecuencia a la falta de actividad física, lo que constituye el cuarto factor de riesgo más importante que conduce a la muerte en todo el mundo (6\% de todas las muertes a nivel mundial), superado solo por la hipertensión (13\%), el consumo de tabaco $(9 \%)$ y los niveles altos de glucosa en la sangre (6\%), indicando además, la misma entidad, que el $60 \%$ de la población mundial no realiza una actividad física adecuada ${ }^{3-5}$.

El sedentarismo se ha convertido en un importante problema y por lo tanto, en una prioridad de salud pública en la última década, ya que aumenta el riesgo de enfermedades no transmisibles como, por ejemplo, enfermedad cardiovascular, diabetes 
y dislipidemias ${ }^{6-8}$, más aun considerando los datos que entrega la Encuesta Nacional de Salud (ENS) de Chile 2016-2017, en la cual se estima que 86,7\% de la población es sedentaria9.

El sedentarismo y la inactividad física en Chile representan uno de los problemas de salud que exhibe mayores tasas de prevalencia junto con otros como el sobrepeso y el elevado consumo de sal ${ }^{9}$. Por otra parte, no existe abundante información recopilada en revisiones que aborden tal problemática, por lo cual, resulta una necesidad valiosa el conocer a través de una revisión sistemática los factores que se asocian a esta situación en Chile, constituyendo además un recurso bibliográfico útil al momento de discutir y proponer políticas de salud que apunten a cambios de conducta en la poblacional nacional.

De acuerdo con la situación anteriormente planteada, surge la pregunta: ¿Qué factores se asocian al sedentarismo e inactividad física en Chile según la evidencia científica?

La presente revisión estableció como objetivo, identificar qué factores se asocian al sedentarismo e inactividad física en Chile según la evidencia científica existente a través de una revisión sistemática.

\section{Material y Método}

Este estudio consiste en una revisión sistemática cualitativa, que se centra en sintetizar el conocimiento producido sobre un tema específico $y$, se presenta la evidencia en forma descriptiva, sin análisis estadístico ${ }^{10}$.

El intervalo de tiempo seleccionado para la búsqueda fue entre el año 2009-2019. La elección de la cohorte de tiempo responde a la disminución porcentual del sedentarismo que entrega la ENS en su encuesta 2016-17 (86,7\%) respecto a la del año 2009-10 (88,6\%), la cual indica que no existe una diferencia estadísticamente significativa9. La pregunta de investigación de la presente revisión fue: ¿Qué factores se asocian al sedentarismo e inactividad física en Chile según la evidencia científica?

Los criterios de inclusión establecidos para los artículos fueron: estudios analíticos de corte transversal., en los idioma inglés-español-portugués, realizados en población chilena, publicados en los últimos 10 años (2009-2019) y en texto completo de acceso gratuito. Los criterios de exclusión fueron artículos repetidos.

Los descriptores utilizados para la recolección tanto en la literatura latinoamericana como internacional fueron seleccionados a través de la terminología en salud (DeCS) de la Biblioteca Virtual de Salud (BIREME) y fueron: "Sedentary Lifestyle", "Risk Factors", "Causality”, "Social Determinants of Health" y sus equivalentes en español: "Estilo de Vida Sedentario", "Factores de Riesgo", "Causalidad” y "Determinantes Sociales de la salud". Además, se utilizaron los siguientes descriptores no controlados: "Sedentary", "Modifiable risk factors", "Non-modifiable risk factors", "Determinants of health" y sus equivalentes en español: "Sedentarismo", "Factores de riego modificables" y "Factores de riesgo no modificables". También, se utilizaron los descriptores Mesh: "Sedentary Behavior" and "Social Determinants of Health".

De forma complementaria se utilizó la conjunción de lógica Booleana "and" para la búsqueda en las bases de datos. La búsqueda fue realizada en el Portal del Centro Latinoamericano y del Caribe de Información en Ciencias de la Salud (BIREME) (https://bvsalud.org/es/) y en la base de datos SCIELO (Scientific Electronic Library Online) (http://www.scielo.org/php/index.php) para indagar en literatura latinoamericana y WoS (Web of Science) (https://www.webofknowledge. com/), Scopus (https://www.scopus.com/), Medline (https://www.ncbi.nlm.nih.gov/pubmed/) para indagar en la literatura internacional.

Se obtuvieron 29.752 artículos de los cuales 29.722 fueron excluidos por no cumplir con los criterios de inclusión y 13 por repetición. Posterior a la lectura de los 17 artículos seleccionados, 12 fueron excluidos por no tratar sobre el tema de los factores que se asocian al sedentarismo e inactividad física en Chile quedando un total final de 5 artículos utilizados para revisión (Figura 1). La búsqueda y análisis crítico de la literatura para la posterior selección de artículos se realizó entre el 8 abril y el 8 mayo de 2019 .

\section{Resultados}

Se puede visualizar en la Figura 1 los descriptores utilizados en cada base de datos, además, del número de artículos encontrados y seleccionados en cada una de ellos. 


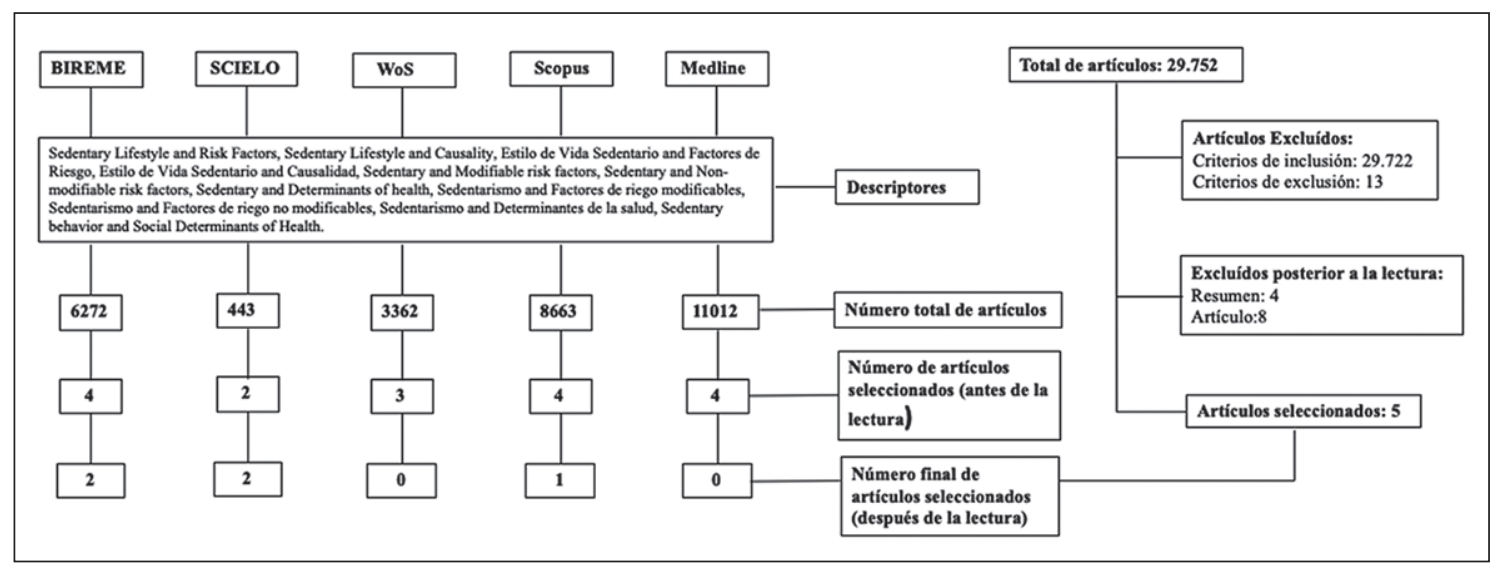

Figura 1. Diagrama de flujo de las bases de datos, descriptores y número de artículos seleccionados.

De forma complementaria, en la Tabla 1 se puede observar las características de los estudios según categoría, país, idioma y revista en la cual fueron publicados. Todos los artículos seleccionados corresponden a estudios originales realizados en Chile y publicados en la Revista Médica de Chile tanto en idioma inglés como español. Hubo tres estudios que se basaron en la ENS 2009-2010 y dos estudios se realizaron con muestras independien- tes, trabajando uno con adultos mayores y el otro con estudiantes universitarios.

A partir del análisis de los artículos seleccionados se estableció tres categorías de asociación: factores asociados al sedentarismo, factores asociados a inactividad física y asociaciones entre actividad física y otros factores. La Figura 2 muestra un resumen de los factores asociados tanto al sedentarismo como a la inactividad física y cuáles a ambos.

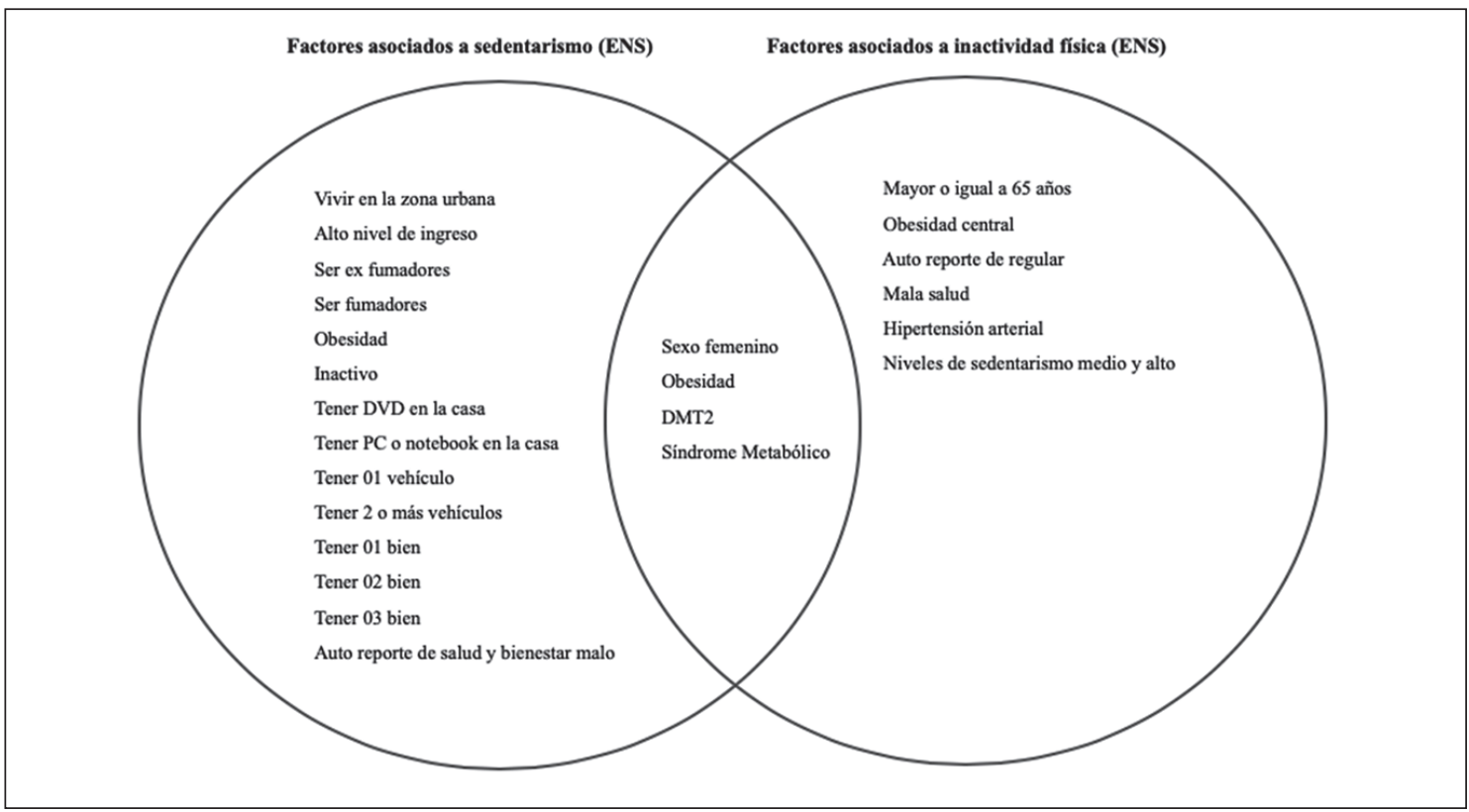

Figura 2. Diagrama de los factores asociados al sedentarismo, a la inactividad física y a ambos. 


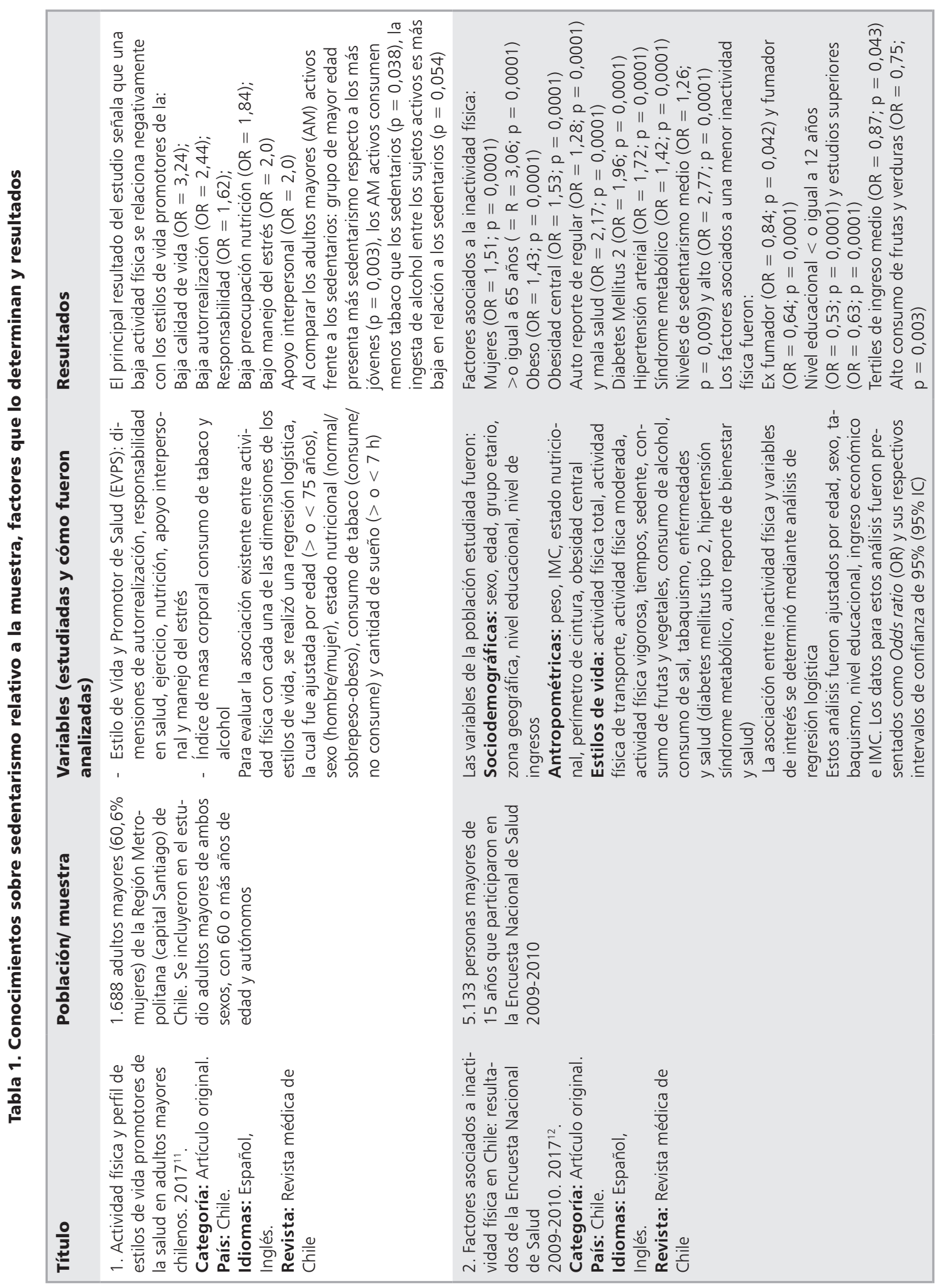




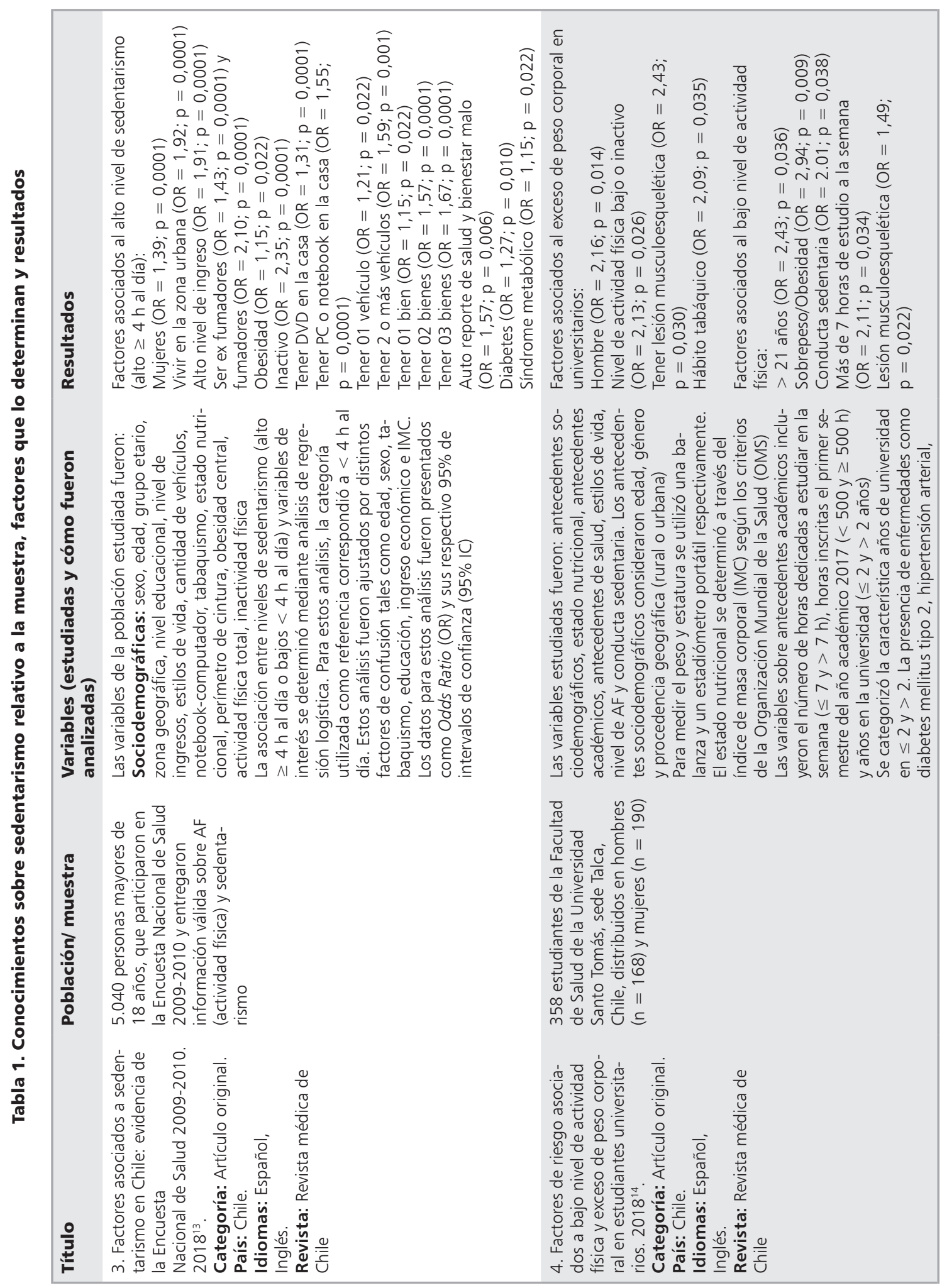




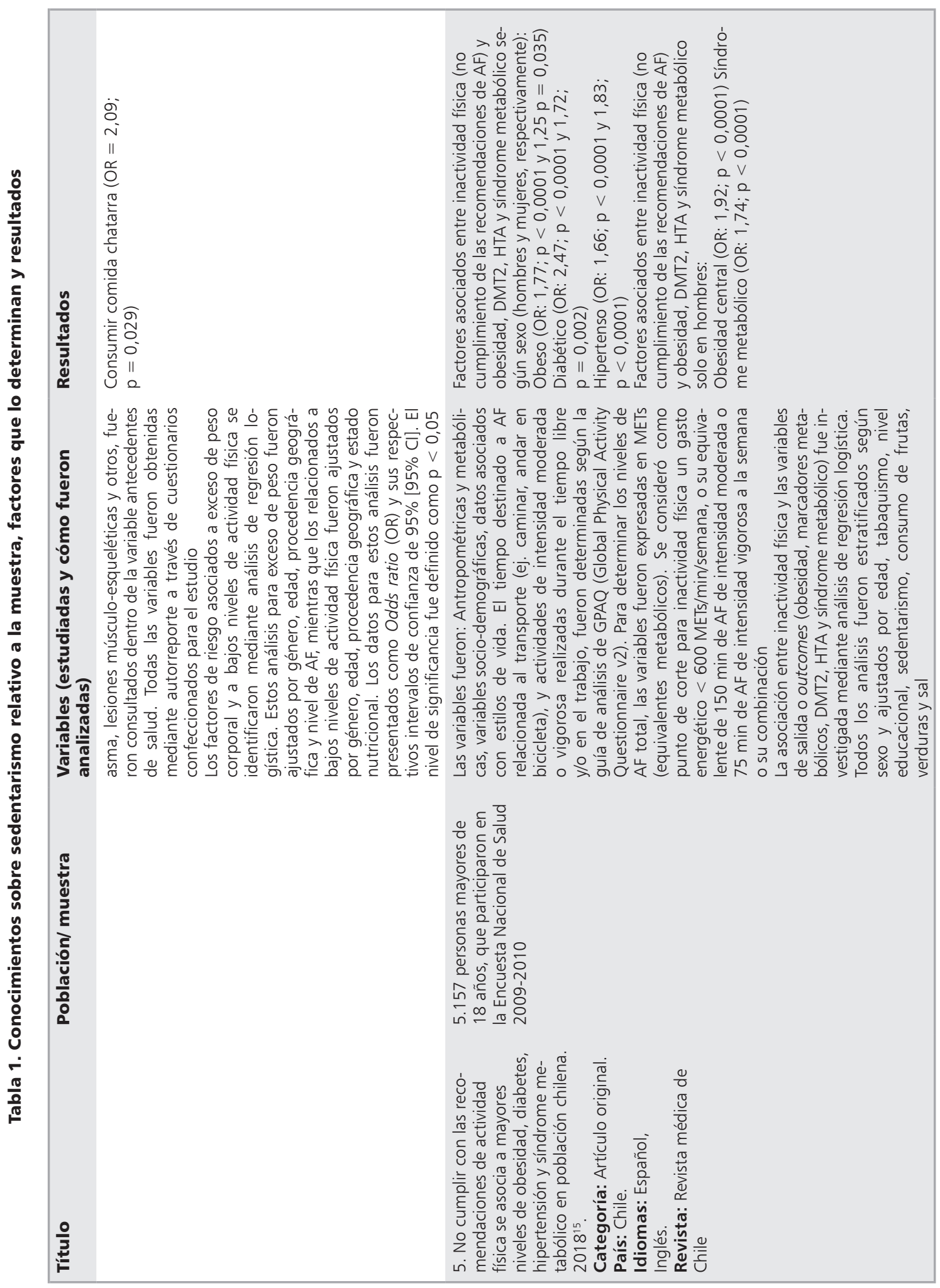


En cuanto a los factores asociados al sedentarismo, los estudios evaluados encontraron las variables sexo femenino, vivir en la zona urbana, alto nivel de ingreso, ser ex fumadores y fumadores, obesidad, inactivo, tener DVD, PC o notebook en la casa, auto reporte de salud y bienestar malo, diabetes mellitus 2 y síndrome metabólico. Además, el estudio llevado cabo en adultos mayores reveló que al comparar los grupos de los activos frente a los sedentarios, el grupo de mayor edad presentó más sedentarismo respecto a los más jóvenes.

Los factores asociados a la inactividad física fueron: sexo femenino, edad mayor o igual a 65 años, obeso, auto reporte de regular y mala salud, diabetes mellitus 2, hipertensión arterial, síndrome metabólico y niveles de sedentarismo medio y alto. Además, el estudio realizado en estudiantes universitarios en Talca, reveló que los factores asociados al bajo nivel de actividad física fueron: ser mayor de 21 años, presentar sobrepeso/ obesidad, conducta sedentaria, dedicar más de 7 horas de estudio a la semana, presentar lesión musculoesquelética y consumir comida chatarra.

\section{Discusión}

De los estudios seleccionados para la revisión, hay que destacar que tres de ellos llevaron a cabo sus análisis considerando la muestra (sobre 5000 participantes) de la ENS 2009-2010, la cual corresponde a un estudio de prevalencia realizado en hogares en una muestra nacional, probabilística y estratificada con representatividad nacional, regional y área urbano/rural chilena ${ }^{12,13,15}$. Otro de los estudios consideró una población muestral de adultos mayores (1.688 participantes) de la Región Metropolitana de Chile ${ }^{11} y$, el último, se efectúo en una población de estudiantes (358 participantes) en una universidad de la ciudad de Talca, Chile ${ }^{14}$.

En cuanto a la inactividad física, la prevalencia en la población chilena aumentó con la edad, fue más alta en adultos con bajo nivel educacional o ingresos, en sexo femenino y en el entorno urbano ${ }^{12,13,15,16 \text {. }}$

Por otra parte, la población chilena muestra una prevalencia de inactividad física (no cumplir con las pautas de actividad física, esto es: $150 \mathrm{~min}$ de actividad física moderada a vigorosa por semana) $(19,8 \%)$ más baja que la estimada en todo el mundo, según el informe descrito por un estudio del año 2012 que estableció el nivel de actividad física en todo el mundo con datos para adultos (15 años o más) de 122 países y para adolescentes (13-15 años) de 105 países ${ }^{1}$.

Además, los datos de prevalencia son similar a los países de Latinoamérica y el Caribe que experimentan una transición epidemiológica (Paraguay $20,3 \%$, Ecuador 22,6\%, Perú 24,5\%, Uruguay $26,6 \%$, Brasil 27,8\% y México 16,8 \%) $)^{1,2}$.

La mayor prevalencia de inactividad física observada en adultos con bajo nivel de educación o ingresos, contrasta con lo reportado en estudios previos realizados en México y Brasil donde un estado socioeconómico más alto se asoció con una mayor prevalencia de inactividad física en comparación con el estado más bajo ${ }^{17,18}$.

En relación al sedentarismo, los tres estudios basados en la ENS 2009-2010, consideraron variables sociodemográficas, antropométricas y de estilos de vida, y en ellos, existe coincidencia respecto a que los principales factores asociados a sedentarismo son el sexo, Índice de Masa Corpórea (IMC), enfermedades crónicas (diabetes mellitus 2, síndrome metabólico), nivel educacional e ingresos ${ }^{12-13,15}$.

El aumento del sedentarismo está fuertemente asociado con un mayor riesgo de enfermedades crónicas no transmisibles y mortalidad, independientemente de los niveles de actividad física ${ }^{19-22}$.

En una revisión sistemática del año 2012 de estudios longitudinales publicados entre 1996 y 2011, se destaca la evidencia que relaciona el tiempo pasado en conductas sedentarias con resultados de salud deficientes en adultos (riesgo cardio-metabólico, diabetes tipo 2 y mortalidad prematura), donde se señala que las asociaciones de comportamiento sedentario con estos resultados adversos persisten incluso cuando se ha tenido en cuenta la participación en actividades físicas de tiempo libre de moderadas a vigorosas ${ }^{20}$.

En otro estudio realizado en EEUU en 2012 donde, se evaluó la cantidad de tiempo empleado en conductas sedentarias y su relación con mortalidad en adultos de 50-71 años, se concluyó que los participantes que reportaron la mayor cantidad de tiempo empleado en ver programas de televisión ( $\geq 7$ h en comparación con $<1$ h/d) tuvieron mayor riesgo de enfermedad cardiovascular y mortalidad por cáncer ${ }^{21}$. En la misma línea, en una revisión del año 2010, se indica que al menos tres estudios en Europa y Australia han examina- 
do las asociaciones de tiempo sedentario medido objetivamente con biomarcadores cardio-metabólicos (observándose asociaciones perjudiciales del tiempo sedentario con la circunferencia de cintura, niveles de triglicéridos, de insulina y de glucosa plasmática ${ }^{22}$.

En Chile, la prevalencia de comportamiento sedentario (tiempo $\geq 4 \mathrm{~h}$ al día destinado a estar sentado) es ligeramente menor $(35,9 \%)$ que la reportada en todo el mundo (41,5\%) (20) y para Argentina (52,8\%), pero es más alta que la reportada para Brasil $(28,2 \%)$ y Colombia $(27,2 \%)^{23}$.

La prevalencia de conductas de riesgo sedentarias por educación y nivel de ingresos en Chile concuerda con las de Brasil, donde los adultos con educación superior e ingresos pasan más tiempo sentados ${ }^{24}$.

La actividad física regular puede ser considerada como uno de los elementos más importantes para la salud de las personas, especialmente, en adultos mayores, puesto que, favorece la reducción de factores de riesgo generales y de mortalidad. En este sentido, los resultados del estudio en adultos mayores realizado en Santiago de Chile el año $2017^{11}$ son coincidentes con trabajos previos, entre los cuales destaca un metaanálisis para evaluar los efectos antidepresivos del ejercicio en adultos mayores, utilizando datos de ensayos controlados aleatorios del año 2016 y otro metaanálisis donde, se investiga los beneficios inducidos por el ejercicio en aspectos específicos de la función ejecutiva en diferentes subgrupos de edad sobre la base de datos de 40 estudios experimentales. En ambos, se concluye que los adultos mayores que ostentan mayor actividad física, poseen también, más probabilidades de presentar un mejor estilo de vida ${ }^{25,26}$.

Estudios han reportado los efectos adversos del sedentarismo sobre la salud, condición que ha surgido como un factor de riesgo potencial, incluso cuando ajustado a los niveles de actividad física que realiza un individuo ${ }^{27,28}$.

Los resultados obtenidos respecto al objetivo de esta investigación, permite concluir que los principales factores corresponden a variables sociodemográficas, antropométricas-metabólicas y estilos de vida. Además, pueden servir de reflexión a los principales actores responsables de establecer políticas de salud pública, las cuales, podrían focalizarse en aquellos grupos que tienen una mayor probabilidad de ser inactivos, lo que podría ayudar a cumplir con las metas sanitarias planteadas para disminuir los niveles de sedentarismo e inactividad física y riesgo asociado a enfermedades cardiovasculares.

No obstante, esta revisión presenta algunas limitaciones tales como el tamaño de la muestra de estudios seleccionados, así como también analizar otros factores como los psicológicos e interpersonales de la población estudiada.

\section{Referencias}

1. Hallal P, Andersen L, Bull F, Guthold R, Haskell W, Ekelund U. Global physical activity levels: surveillance progress, pitfalls, and prospects. Lancet 2012; 380 (9838): 247-57.

2. Guthold R, Ono T, Strong K Chatterji S, Morabia A. Worldwide variability in physical inactivity a 51-country survey. Am J Prev Med 2008; 34 (6): 86-94.

3. World Health Organization. Global health risks: mortality and burden of disease attributable to selected major risks. Geneva: World Health Organization; 2009; Pag: 1-70. Disponible en: https://www.who.int/healthinfo/ global_burden_disease/GlobalHealthRisks_report_full. pdf [Consultado el 11 de mayo de 2019].

4. Kohl H, Craig C, Lambert E, Inoue S, Alkandari J, Leetongin $\mathrm{G}$ et al. The pandemic of physical inactivity: Global action for public health. The Lancet 2012; 380 (9838): 294-305.

5. $\mathrm{Hu} \mathrm{B}$, Sedentary lifestyle and risk of obesity and type 2 diabetes. Lipids 2003; 38 (2): 103-8.

6. Jorquera C, Cancino J. Exercise, obesity and metabolic síndrome. Rev Med Clin Condes 2012; 23 (3): 227-35.

7. Buck C, Loyen A, Foraita R, Van Cauwenberg J, De Craemer M, Mac Donncha C. Factors influencing sedentary behaviour: A system based analysis using Bayesian networks within DEDIPAC. PLoS One 2019; 14 (1): 1-18.

8. Kotlarczyk M, Hergenroeder A, Gibbs B, Cameron F, Hamm M, Brach J. Personal, social, and environmental factors influencing sedentary behavior in long-term care community residents. Innovation in Aging 2018; 2 (1): 567.

9. MINSAL. Encuesta Nacional de Salud 2016-2017. Departamento de Epidemiología, División de Planifiación Sanitaria, Subsecretaría de Salud Pública 2017. Pag: 1-61. Disponible en: https://www.minsal.cl/wp-content/ uploads/2017/11/ENS-2016-17_PRIMEROS-RESULTADOS.pdf [Consultado el 15 de mayo de 2019].

10. Letelier SLM, Manríquez MJJ, Gabriel Rada GG. Revi- 
siones sistemáticas y metaanálisis: ¿son la mejor evidencia? Boletín de la Escuela de Medicina 2005; 30 (2): 37-9.

11. Durán S, Sánchez H, Valladares M, López A, Valdés, $\mathrm{P}$, Herrera T. Actividad física y perfil de estilos de vida promotores de la salud en adultos mayores chilenos. Rev Med Chile 2017; 145: 1535-40.

12. Díaz-Martínez X, Garrido A, Martínez M, Leiva A, Álvarez C, Ramírez-Campillo R. Factores asociados a inactividad física en Chile: resultados de la Encuesta Nacional de Salud 2009-2010. Rev Med Chile 2017; 145 (10): 1259-67.

13. Martínez M, Leiva A, Petermann F, Garrido A, Díaz X, Álvarez C, et. al. Factores asociados a sedentarismo en Chile: evidencia de la Encuesta Nacional de Salud 20092010. Rev Med Chile 2018; 146 (1): 22-31.

14. Concha Y, Guzmán E, Valdés P, Lira C, Petermann F, Celis C. Factores de riesgo asociados a bajo nivel de actividad física y exceso de peso corporal en estudiantes universitarios. Rev Med Chile 2018; 146 (8): 840-9.

15. Díaz-Martínez X, Petermann F, Leiva A, Garrido A, Salas C, Martínez M. No cumplir con las recomendaciones de actividad física se asocia a mayores niveles de obesidad, diabetes, hipertensión y síndrome metabólico en población chilena. Rev Med Chile 2018; 146 (5): 58595.

16. Celis C, Salas C, Alduhishy A, Sanzana R, Martínez A, Leiva A. Socio-demographic patterns of physical activity and sedentary behaviour in Chile: results from the $\mathrm{Na}$ tional Health Survey 2009-2010. J Public Health 2016; 38 (2): 98-105.

17. Medina C, Janssen I, Campos I, Barquera S. Physical inactivity prevalence and trends among Mexican adults: results from the National Health and Nutrition Survey (ENSANUT) 2006 and 2012. BMC Public Health 2013; 13: 1063.

18. Hallal P, Victoria C, Wells J, Lima R. Physical inactivity: prevalence and associated variables in Brazilian adults. Med Sci Sports Exerc 2003; 35 (11): 1894-900.

19. Bennie J, Chau J, van der Ploeg H, Stamatakis E, Do A, Bauman A. The prevalence and correlates of sitting in European adults - a comparison of 32 Eurobarometer-participating countries. Int J Behav Nutr Phys Act 2013; 10 (107): 1-13.

20. Dunstan D, Howard B, Healy G, Owen N. Too much sitting - a health hazard. Diabetes Res Clin Pract 2012; 97 (3): 368-76.

21. Matthews C, George S, Moore S, Heather R, Blair A, Park Y et al. Amount of time spent in sedentary behaviors and cause-specific mortality in US adults. Am J Clin Nutr 2012; 95 (2): 437-45.

22. Owen N, Healy G, Matthews C, Dunstan D. Too much sitting: the population health science of sedentary behavior. Exerc Sport Sci Rev 2010; 38 (3): 105-13.

23. Bauman A, Ainsworth B, Sallis J, Hagströmer M, Craig C, Bull F. The descriptive epidemiology of sitting. A 20-country comparison using the International Physical Activity Questionnaire (IPAQ). Am J Prev Med 2011; 41 (2): 228-35.

24. Suzuki C, de Moraes S, de Freitas I. Sitting-time means and correlates in adults living in Ribeirao Preto-SP, Brazil, in 2006: OBEDIARP project. Rev Bras Epidemiol 2010; 13 (4): 699-712.

25. Schuch F, Vancampfort D, Rosenbaum S, Richards J, Ward P, Veronese N, et al. Exercise for depression in older adults: a meta-analysis of randomized controlled trials adjusting for publication bias. Rev Bras Psiquiatr 2016; 38 (3): 247-54.

26. Ludyga S, Gerber M, Brand S, Holsboer-Trachsler E, Pühse U. Acute effects of moderate aerobic exercise on specific aspects of executive function in different age and fitness groups: A meta-analysis. Psychophysiology 2016; 53 (11): 1611-26.

27. Honda T, Chen S, Yonemoto K, Kishimoto H, Chen T, Narazaki K, et al. Sedentary bout durations and metabolic syndrome among working adults: a prospective cohort study. BMC Public Health 2016; 16: 888.

28. Koster A, Caserotti P, Patel KV, Matthews CE, Berrigan D, Van Domelen DR, et al. Association of sedentary time with mortality independent of moderate to vigorou physical activity. PloS one 2012; 7 (6): e37696. 